\title{
MiR-122 in hepatic function and liver diseases
}

\author{
Jun Hu, Yaxing Xu, Junli Hao, Saifeng Wang, Changfei Li, Songdong Meng \\ CAS Key Laboratory of Pathogenic Microbiology and Immunology, Institute of Microbiology, \\ Chinese Academy of Sciences (CAS), Beijing 100101, China \\ $\bowtie$ Correspondence: mengsd@im.ac.cn \\ Received February 5, 2012 Accepted February 22, 2012
}

\begin{abstract}
As the most abundant liver-specific microRNA, microRNA-122 (miR-122) is involved in various physiological processes in hepatic function as well as in liver pathology. There is now compelling evidence that miR122 , as a regulator of gene networks and pathways in hepatocytes, plays a central role in diverse aspects of hepatic function and in the progress of liver diseases. This liver-enriched transcription factors-regulated miRNA promotes differentiation of hepatocytes and regulates lipid metabolism. With regard to liver diseases, miR-122 was shown to stimulate hepatitis C virus (HCV) replication through a unique and unusual interaction with two binding sites in the 5'-UTR of HCV genome to mediate the stability of the viral RNA, whereas inhibit the expression and replication of hepatitis $B$ virus (HBV) by a miR-122-cylin G1/p53-HBV enhancer regulatory pathway. In addition, miR-122 acts as a suppressor of cell proliferation and malignant transformation of hepatocytes with remarkable tumor inhibition activity. Notably, a clinical trial targeting miR-122 with the anti-miR-122 oligonucleotides miravirsen, the first miRNA targeted drug, has been initiated for treatment of HCV infection. With further understanding of the comprehensive roles of miR-122 in hepatic functions and the mechanisms involved in miR-122 down-regulation in chronic hepatitis or hepatocellular carcinoma, miR-122 appears to be a promising candidate for effective therapeutic approaches against tumor and infectious diseases.
\end{abstract}

KEYWORDS miR-122, liver development, lipid metabolism, hepatitis $\mathrm{C}$ virus (HCV), hepatitis B virus (HBV), hepatocellular carcinoma ( $\mathrm{HCC})$

\section{INTRODUCTION}

Since the discovery of microRNA (miRNA) lin-4 in Caenor- habditis elegans, especially the subsequent identification of another miRNA let-7 as a new posttranscriptional regulator of gene expression more than a decade ago (Fire et al., 1998; Reinhart et al., 2000), the small non-coding RNA known as miRNA has been extensively studied in plants, animals and human beings. miRNAs are a large class of small non-coding and double-stranded RNA molecules of approximately 22 nucleotides in length. Full-length miRNAs are transcripted by RNA polymerase II, which are called pri-miRNAs (hairpin precursors). These pri-miRNAs are processed by Drosha within the nuclear compartment to produce pre-miRNAs (hairpins) of about 65 nucleotides in length, which are transported into the cytoplasm and further cleaved by Dicer to produce their mature form miRNAs of approximately 22 nucleotides in length (Lindsay, 2008; Newman and Hammond, 2010). Mature miRNA duplexes are loaded onto the RNA-induced silencing complex (RISC) which consists of a member of the double-stranded RNA binding protein argonaute family (Ago). Using the guide strand of the miRNA, the RISC recognizes and interacts with conserved complementary target sites in target mRNAs (often in the $3^{\prime}-$ UTR) through canonical base-paring between the seed region of nucleotides about 6-8mer, and induces their translational repression, deadenylation and degradation (Miyoshi et al., 2009; Rusca and Monticelli, 2011).

So far, more than 1500 human miRNAs (has-mir) and 700 mouse miRNAs (mmu-mir) have been identified and described at the miRBase website (http://www.mirbase.org, released in Jan 2012). As most miRNAs guide the recognition of imperfect matches of target mRNAs and regulate their expression at posttranscriptional levels, individual miRNAs have multiple or even up to tens of mRNA targets, indicating that miRNAs may have a similar role to transcription factors (Lewis et al., 2005). Indeed, it is estimated that more than one third of human protein-coding genes are subjected to modulation by miRNAs (Sage et al., 2011). In addition, miRNA expression profiling studies revealed that these tiny gene 
expression regulators exhibit limited developmental stage-, tissue or cell type-, and disease-specific patterns, although some miRNAs are widely expressed (Carissimi et al., 2009). In conditional Dicer knockout mice which provide a model to determine miRNA function, miRNAs have been shown to be involved in various physiological processes, including development (Murchison et al., 2007), immunity (Belver et al., 2010), differentiation (Zhang et al., 2010) and homeostasis (Zhou et al., 2008). Moreover, recent studies have provided compelling evidence that deregulation of miRNAs has resulted in human diseases, such as autoimmune diseases and cancer (Starczynowski et al., 2010; Kasinski and Slack, 2011; Luo et al., 2011).

In this study we focus on recent advance in the study of the most abundant liver-specific microRNA miR-122 in hepatic function and liver pathology, as miR-122 is a typical example showing how a single miRNA mediates the expression of multiple target genes, the signaling pathways and regulatory networks.

\section{MIR-122 IN LIVER DEVELOPMENT}

It is well documented that vertebrate miR-122 is a liver-specific miRNA which is expressed almost solely in hepatocytes at above 50,000 copies per cell (Filipowicz and Grosshans, 2011), although a recent study has shown that miR-122 is also present in human skin fibroblasts, the stability or activity of which may be controlled by the noncanonical poly(A) polymerase Gld2 (Burns et al., 2011). As a liver-specific miRNA, miR-122 reaches approximately $70 \%$ of the total miRNA population in the adult liver, and notably, its expression is sharply up-regulated in both mouse and human liver during embryonic development (Girard et al., 2008). These observations suggest that miR-122 may play an essential role in the regulation of hepatocyte differentiation and liver development. Direct evidence that miR-122 might be involved in liver development was first provided by Xu et al. (2010), who showed in mice that four liver-enriched transcription factors including hepatocyte nuclear factor (HNF) $1 \alpha, H N F 3 \beta$, HNF4 $\alpha$ and CCAAT/enhancer-binding protein (C/EBP) $\alpha$ bind to miR-122 promoter and activate miR-122 expression. The increase of miR-122 leads to down-regulation of its target CUTL1, a transcriptional repressor of genes specifying terminal differentiation in hepatocytes, thereby contributing to differentiation of hepatocytes (Xu et al., 2010). A subsequent study further showed that as transcriptional stimulators, HNF6 and its paralog Onecut2 promote the expression of miR-122 which is required for proper progression of hepatocyte differentiation. Significantly, miR-122 was also found to stimulate HNF6 expression. Thus miR-122 and HNF6 establish a positive feedback loop involved in directing hepatocyte differentiation and liver development (Laudadio et al., 2012). Together, the most abundant liver-specific microRNA miR-122, and liver-enriched transcription factors scription factors such as HNFs, have an integral role in liver development and function.

\section{MIR-122 IN LIPID METABOLISM}

Historically, miR-122 is the first miRNA identified to regulate lipid metabolism (Filipowicz and Grosshans, 2011). Due to its location and abundance in the liver, miR-122 is proposed to be directly involved in cholesterol accumulation and fatty acid metabolism. There are a number of mouse models and nonhuman primates that have been studied to silence miR-122. With antisense strategies, miR-122 sequestration by its antagomir leads to decreased cholesterol levels and low density lipoprotein (LDL) and high density lipoprotein (HDL) fractions both in the liver and blood, and decreases liver fat accumulation in the liver (Krützfeldt et al., 2005; Esau et al., 2006; Elmén et al., 2008a, b; Esau, 2008). Notably, the silencing of miR-122 by antisense targeting in high-fat fed mice has been shown to lead to long-lasting reduction of hepatic steatosis with decreased cholesterol synthesis rates and enhanced hepatic fatty-acid oxidation, making miR-122 a potential target for the treatment of dyslipidemias.

Despite these achievements in studies of miR-122-mediated lipid metabolism, the molecular mechanisms underlying miR-122-regulated cholesterol biosynthesis remain elusive and still await further investigation (Moore et al., 2010). As now no direct gene targets of miR-122 have been identified which are involved in cholesterol metabolism, it is possible that miR-122 acts indirectly. Nevertheless, Norman and Sarnow (2010) proposed that miR-122 may regulate an inhibitor of the mevalonate pathway which functions to synthesize cholesterol and isoprenoid intermediates. Furthermore, several genes, which are involved in fatty acid synthesis and oxidation, have been shown to be subjected to regulation by miR-122 (Moore et al., 2010). More studies are needed to understand the mechanisms of gp96 in the regulation of lipid metabolism, as well as to address the clinical relevance of miR-122 and its role in disorders of lipid metabolism by comprehensive miR-122 profiling and functional analysis.

\section{MIR-122 IN HCV INFECTION}

The role of miR-122 in controlling HCV (hepatitis C virus) infection, including its stimulation of the replication and expression of $\mathrm{HCV}$ in an unusual manner, represents a major effort in this field. Around 180 million people worldwide are chronically infected with $\mathrm{HCV}$, a strictly hepatotropic positive-sense RNA virus which causes fatal liver diseases. According to phylogenetic analyses, $\mathrm{HCV}$ is currently classified into seven major genotypes and numerous subtypes. The $\mathrm{HCV}$ genome is around $9.6 \mathrm{~kb}$ in length, comprising a single ORF flanked by 5'- and 3'-UTRs (Filipowicz and Grosshans, 2011). Currently, there is no vaccine yet for hepatitis $C$, and chronic hepatitis $\mathrm{C}(\mathrm{CHC})$ is a disease that has a huge impact 
on global public health, as it is correlated with a significantly increased risk for the development of cirrhosis and hepatocellular carcinoma (HCC).

Viruses that cause chronic infection, including HCV, are notorious for their ability to evade host defense and usurp cellular pathways for the establishment of persistent infection. The first indication that miR-122 is involved in regulating $\mathrm{HCV}$ RNA abundance came from Jopling and colleagues (2005) that miR-122 is likely to facilitate viral RNA replication. They found two miR-122 binding sites in viral mRNA, which are located within the $5^{\prime}$-noncoding region (NCR), only $21 \mathrm{nt}$ from the $5^{\prime}$ end of the viral genome, and within the $3^{\prime}-\mathrm{NCR}$, respectively. Their further study showed that sequestration of miR-122 leads to a marked loss of replicating viral RNAs, and simultaneous recognition of the binding site within 5'-NCR by miR-122 is required for miR-122-induced viral RNA accumulation, suggesting that miR-122 is likely to facilitate viral RNA replication through interaction with viral 5 '-NCR. Since then a large number of impressive experiments have been performed primarily on structure and functional analyses that support that the interaction between miR-122 and viral $5^{\prime}$ NCR is essential to promote HCV replication. Chang et al. (2008) showed that miR-122 could also enhance HCV replication in nonhepatic human embryonic kidney epithelial cells (HEK-293). Interestingly, expression of miR-122 has been shown to endow the ability of supporting efficient HCV RNA replication and infectious virion production in HepG2 cells (Narbus et al., 2011). Henke et al. (2008) further uncovered a unique interaction of miR-122 with two binding sites in the 5'-UTR of the viral genome, which stimulates HCV translation by enhancing the association of ribosomes with the viral RNA. Structure analysis demonstrated that miR-122 triggers an open conformation of the HCV internal ribosome entry site (IRES), which may facilitate HCV translation (Díaz-Toledano et al., 2009). Adding to the complexity of miR-122 regulatory role in HCV replication, it is also suggested that besides binding to both sites of viral 5'-UTR, miR-122 is likely to affect viral replication at an additional step in the HCV life cycle through comparison of the replication capacities of the double-binding-site mutant and an IRES mutant strain (Jangra et al., 2010). Furthermore, the viral 5'-UTR secondary structure in the presence of miR-122 has been recently resolved, showing that almost all necleotides in miR-122 are involved in binding to the second site (Pang et al., 2012). Finally, Ago2 was also shown to associate with miR-122-HCV 5'-UTR complex, and the RISC-like complex mediates the stability of HCV RNA and protects the viral genome from $5^{\prime}$ exonuclease digestion by host mRNA decay machinery (Shimakami et al., 2012). In this context, the miR-122/RISC complex would therefore act in an unusual way to stabilize viral RNA and decrease its decay, rather than to induce its degradation as most miRNAs do, expanding the knowledge of how miRNAs modulate gene expression in multiple ways.

Somewhat differently from the mechanism mentioned above, a study performed in cell experiment showed that silencing of miR-122 with antagomir decreases HCV RNA abundance whereas transfection of miR-122 mimics increases HCV level. In the meantime, antagomir of miR-122 also up-regulates Heme oxygenase-1 (HO-1) probably via decreasing its transcription repressor Bach1, and HO-1 significantly inhibits HCV replication (Shan et al., 2007). These data suggest that miR-122 promotes HCV replication partly via down-regulation of HO-1. In addition, miR-122 was also shown to be regulated by IFN $\beta$ (Pedersen et al., 2007). In particular, IFN $\beta$-induced reduction of miR-122 may contribute to IFN $\beta$-mediated inhibition of $\mathrm{HCV}$ as miR-122 is required for efficient HCV replication, supporting the notion that through the interferon system, the host may use cellular miRNAs against viral infections.

The demonstration from the extensive experiments mentioned above that miR-122 is essential for efficient HCV replication provides the potential of targeting miR-122 as an effective strategy to either limit HCV infection or prevent $\mathrm{CHC}$-induced HCC. Indeed, recently Lanford and colleagues (2010) reported that miR-122 silencing in chronically HCV infected chimpanzees using a locked nucleic acid (LNA)-modified phosphorothioate oligonucleotide complementary to the $5^{\prime}$ end of miR-122 leads to potent and sustained inhibition of HCV replication. Antagomir of miR-122 decreases free miR-122 levels in the liver by more than 300 fold determined by real-time PCR, and accordingly results in a maximum decrease of 2.6 and 2.3 orders of magnitude in HCV RNA levels in the serum and the liver, respectively. Moreover, elevated alanine aminotransferase (ALT) is reduced to normal levels during therapy, which may be due to reduction of viral loads. Notably, no apparent viral resistance was observed during treatment with the miR-122 antagomir, as shown by the lack of adaptive mutations in the two miR-122 seed sites of viral 5'-UTR. Finally, the authors provided convincing evidence of the feasibility and safety of the miR-122 antagomir in the nonhuman primate model.

The conclusions from these elegant studies above would be expected to be confirmed by experiments in humans. However, several unanswered questions remain. For instance, there are insufficient data to determine if miR-122 plays a role in patients with $\mathrm{CHC}$ and whether miR-122 is an ideal target for HCV therapy. Since miR-122 plays an essential role in $\mathrm{HCV}$ replication, the experimental data and mouse and primate models predict that $\mathrm{CHC}$ patients with lower levels of miR-122 should have a higher responding rate to IFN therapy, which is an important first-line treatment option for HCV infection. However, this is not the case. By detection of miR-122 levels in livers of $42 \mathrm{CHC}$ subjects by real-time PCR, Sarasin-Filipowicz et al. (2009) observed that miR-122 levels are significantly lower in subjects with primary nonresponders (PNR) to pegIFN- $\alpha$ therapy than those with complete early virological responders (cEVR). In addition, miR-122 levels were shown to be negatively correlated with 
the expression of IRGs, indicating that $\mathrm{CHC}$ subjects with PNR have both lower pretreatment miR-122 levels and higher IRG levels. Another puzzling feature is that hepatic miR-122 expression in $\mathrm{CHC}$ patients was shown unrelated with the hepatic HCV load, and was inversely correlated with the severity of functional and histopathological liver damage (Morita et al., 2011). This paper therefore states that miR-122 by itself is not a critical molecular target for HCV therapy. Taken together, there are insufficient clinical data to validate miR-122 as a new targeting approach for $\mathrm{CHC}$ therapy. More studies are needed to understand the regulatory role of miR-122 in various states of HCV infection and its use as a targeted molecule for the development of novel molecular-targeted therapies of hepatitis $\mathrm{C}$.

\section{MIR-122 IN HBV INFECTION}

HBV, another main hepatotropic DNA virus, causes chronic infection in about 350 million people worldwide. Chronic hepatitis $B(\mathrm{CHB})$ is caused by HBV infection which has a huge impact on global public health as it is correlated with a significantly increased risk for the development of cirrhosis, liver failure and HCC. However, until now the mechanisms for viral evasion of host defense and pathogenesis of HBV infection are still unclear. Information regarding the balance between viral parameters and host defense factors is essential to understand the molecular basis for viral pathogenesis, which may facilitate the development of novel targeted agents with improved therapeutic efficiencies.

In contrast to the extensive studies on the biological functions of miR-122 in HCV infection, presently only a few studies have addressed this issue in HBV infection. Given the important roles of miR-122 in hepatic function and liver pathology, we examined whether miR-122 affects HBV expression and replication. We found that transfection of a miR-122 mimic inhibits HBV expression, whereas antisense inhibition of miR-122 leads to increased HBV production in transfected cells. The result indicates that in contrast to $\mathrm{HCV}$, the most abundant liver-specific miRNA miR-122 actually inhibits replication of the hepatotropic virus HBV, suggesting that therapies that increase miR-122 may be an effective strategy to limit HBV replication. Further study showed that down-regulation of $\mathrm{HO}-1$ by $\mathrm{miR}-122$ plays a negative role in miR-122-mediated inhibition of viral expression. Several lines of evidence also confirmed the inhibition effect of miR-122 on HBV (Chen et al., 2007, 2011; Fan et al., 2011). These studies showed that besides acting as a suppressor of cell proliferation and malignant transformation of hepatocytes, miR-122 significantly inhibits HBV expression and replication. Hepatitis $B$ is mainly transmitted through vertical infection at birth or horizontal transmission. The majority of adult patients recover completely from their HBV infection while the others will become chronically infected hepatitis. Several host fac- tors, including the HLA alleles, IFN and interleukins, have been shown to influence the clinical outcome or disease severity (Kummee et al., 2007; Ramezani et al., 2008; Tan et al., 2008). However, until now factors determining the outcome of HBV infection have not been fully understood. Conceivably, the pronounced inhibitory effect of miR-122 on HBV may shape the virus-host interactions due to the differential expression of miR-122 among healthy individuals.

Based on these earlier studies above mentioned demonstrating miR-122 suppresses HBV expression and replication, we then explored the mechanisms of miR-122-mediated HBV inhibition and searched for miR-122 target mRNAs that might play a role in HBV replication. We identified cyclin $\mathrm{G} 1$ as a miR-122 target from multiple candidate target genes which was shown to be involved in the regulation of HBV replication. Overexpression and knockdown studies both showed that cyclin G1 repressed viral replication in HBV transfected cells. Further study revealed that miR-122 down-regulates its target cyclin G1, thus interrupts interaction between cyclin G1 and p53, and enhances p53-mediated inhibition of HBV replication by allowing the specific binding of p53 to HBV enhancer elements and thereby enhancing p53-mediated inhibition of HBV transcription. These results revealed the mechanism of miR-122-mediated HBV suppression by modulation of p53 through the down-regulation of cyclin $\mathrm{G} 1$ or/and promotion of p53 activity through direct or indirect binding to cyclin G1 (Wang et al., 2012).

An intriguing question arises from the above studies is why the hepatotropic virus HBV seems to live in the liver "happily" while the highly expressed and basically liver-specific miR-122 actually suppresses its expression and replication. Our preliminary observation showed that miR-122 expression in the liver is significantly decreased in patients with HBV infection compared with healthy controls, and the miR-122 levels are negatively associated with intrahepatic viral loads in CHB (Wang et al., 2012). We therefore speculate that under chronic HBV infection the virus may interact with its host hepatocytes to facilitate its replication and pathogenesis by down-regulation of miR-122. This may help to understand how HBV has evolved strategies to establish conditions favoring viral replication and persistence. It is also worthwhile to explore the mechanisms involved in miR-122 down-regulation by HBV infection. Until now few studies have addressed this issue. Pedersen et al. (2007) have shown that IFNs, which are usually up-regulated in $\mathrm{CHB}$, lead to a significant decrease in the expression of miR-122 both in vitro and in vivo. We also observed a marked decrease (about $50 \%$ at maximum) of the expression of miR-122 under IFN- $\alpha$ treatment in Huh-7 cells. Since the expression of miR-122 has been shown to be transcriptionally regulated by liver-enriched transcription factors (Xu et al., 2010), analysis of the regulation of miR-122 expression by these transcriptional factors is needed. 


\section{MIR-122 IN HCC}

There is growing literature on biological and clinical significance of miR-122 in the development of HCC. Using a genome-wide expression profiling approach, Burchard et al (2010) have recently shown decreased miR-122 expression and increased miR-122 predicted target genes in tumor tissues compared with nontumor tissues from HCC, which may possibly lead to impairment of mitochondrial metabolism. Loss of miR-122 expression in HCC, which is associated with poor prognosis and metastasis, has been confirmed by several other studies (Coulouarn et al., 2009; Bai et al., 2009; Zeng et al., 2010; Mizuguchi et al., 2011; Karakatsanis et a., 2011). In these studies, miR-122 is shown to be involved in cell proliferation, apoptosis, clonogenic survival, migration, in vivo invasion, and tumor formation in nude mice. Loss of miR-122 facilitates cell tumorigenic properties such as cell migration and invasion, and restoration of miR-122 reverses this phenotype. Currently, several target genes of miR-122 have been identified to be involved in hepatocarcinogenesis, such as ADAM10 (a distintegrin and metalloprotease family 10), serum response factor (SRF) (Bai et al., 2009), insulin-like growth factor 1 receptor (Igf1R) (Zeng et al., 2010), cyclin G1 (Fornari et al., 2009), and Wnt1 (Xu et al., 2012). In addition, overexpression and restoration of miR-122 in HCC cells has been shown to sensitize HCC cells to chemotherapeutic agents (Bai et al., 2009; Xu et al., 2011; Yang et al., 2011). Owing to its remarkable tumor inhibitory activity, increasing miR-122 levels may be a promising strategy for HCC treatment (Ma et al., 2010).

\section{CONCLUSION AND OUTLOOK}

Since its discovery in 2002, miR-122 as the highly expressed and liver-specific microRNA, has been extensively studied concerning its biological functions and clinical significance. Figure 1 illustrates miR-122 as a regulator of gene networks and pathways in hepatocytes. According to this illustration, liver-enriched transcription factors-regulated miR-122 promotes differentiation of hepatocytes, and contributes to hepatic function such as cholesterol synthesis. With regard to liver diseases, miR-122 stimulates HCV replication whereas inhibits replication of $\mathrm{HBV}$, and suppresses hepatocarcinogenesis. Overall, there is now compelling evidence that miR-122 is involved in diverse aspects of hepatic function and modulation of its activity might ultimately provide a novel therapeutic approach in the treatment of hepatitis B and C, as well as HCC.

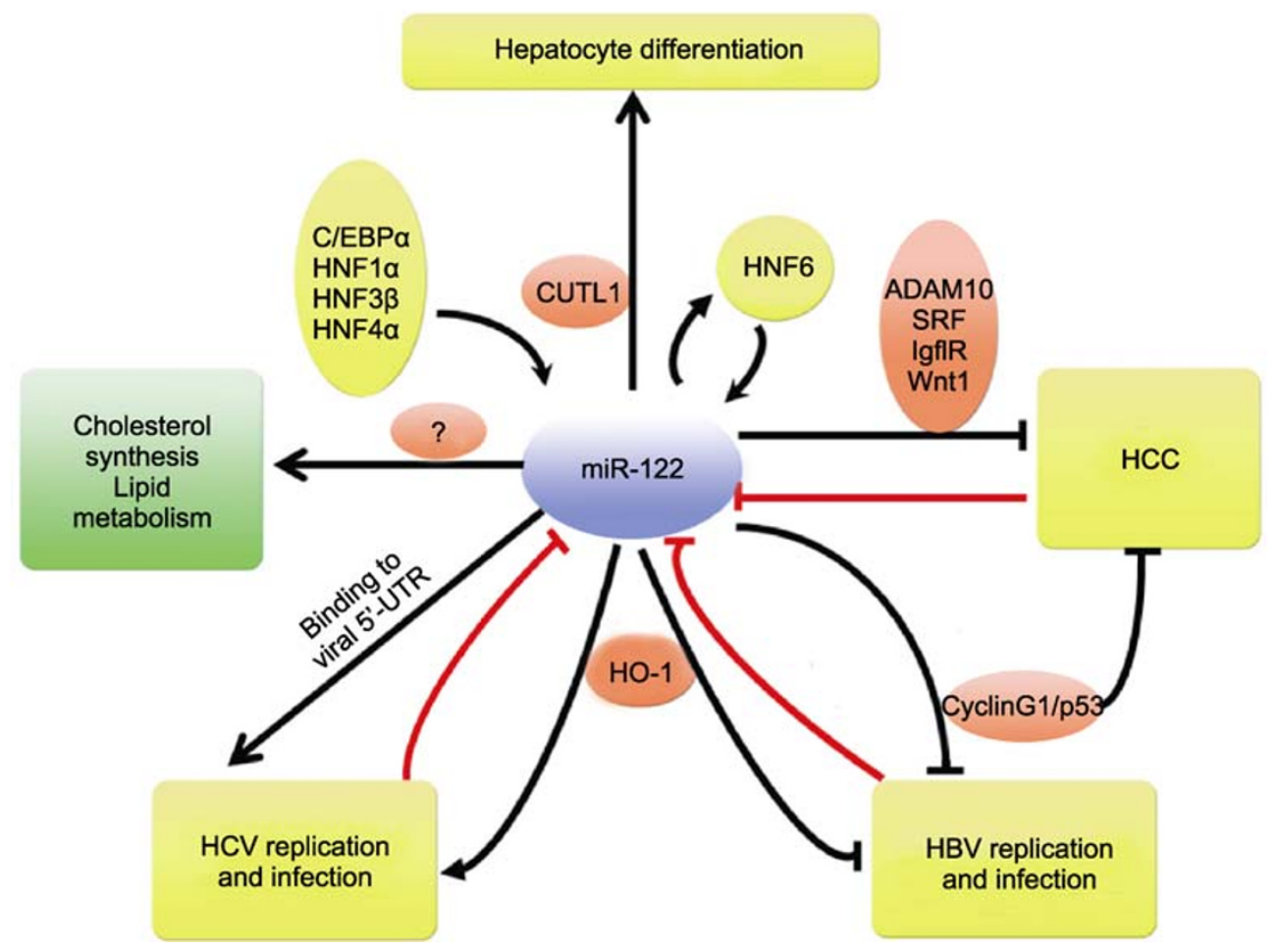

Figure 1. Role of miR-122 in the regulation of hepatic function and liver diseases. The illustration summarizes the current understanding of the network of interactions between miR-122, transcription factors (yellow circles) and its target genes (red circles) for regulation of hepatocyte differentiation, lipid metabolism, HBV and HCV replication, and the development of HCC. Stimulation ( $\uparrow$ ) or inhibition $(T)$ is determined following how miR-122 impacts on the indicated biological responses (green circles) and how these biological responses could in turn influence miR-122 expression (indication in red). 
The finding that miR-122 silencing by anti-miR-122 oligonucleotides leads to potent and sustained inhibition of $\mathrm{HCV}$ replication may have important implications for HCV therapy. Indeed, a clinical trial of targeting miR-122 with miravirsen (SPC3649) utilizing LNA drug platform, the first miRNA targeted drug, has been initiated for treatment of HCV infection (http://www.santaris.com). Data from the Phase 2a clinical study showed that miravirsen treatment is well tolerated, and reduces HCV RNA levels to undetectable levels in patients with $\mathrm{HCV}$, validating its promising potential for $\mathrm{CHC}$ therapy. As miR-122 also plays essential roles in hepatic functions as well as in liver diseases including $\mathrm{CHB}$ and $\mathrm{HCC}$, it will be important to take into consideration the unique features of miR-122 when treating $\mathrm{CHC}$ with the miR-122 inhibitors in the long run.

Dramatic decrease of miR-122 levels in the liver has been observed in both $\mathrm{CHB}$ and $\mathrm{HCC}$, and loss of miR-122 has been suggested to contribute to persistence of viral infection and hepatocarcinogenesis. Conceivably, the activities of miR-122 may be one of the contributing reasons why it has been so difficult to design effective therapies for $\mathrm{CHB}$ and HCC. Mechanistic studies are urgently needed to explore the causes of reduction of miR-122 in CHB and HCC. In addition, given that aberrant expression of miR-122 has been associated with hepatitis B and HCC, it will be important to investigate the potential of increasing the miR-122 level as an effective strategy to either limit HBV infection or prevent the development of HCC.

\section{ACKNOWLEDGEMENTS}

This work was supported by grants from the National Natural Science Foundation of China (Grant Nos. 30970146, 91029724, and 81021003).

\section{ABBREVIATIONS}

ADAM10, a distintegrin and metalloprotease family 10; Ago, argonaute; ALT, alanine aminotransferase; C/EBP, CCAAT/enhancerbinding protein; $c E V R$, complete early virological responders; $\mathrm{CHB}$, chronic hepatitis $\mathrm{B} ; \mathrm{CHC}$, chronic hepatitis $\mathrm{C}$; $\mathrm{HBV}$, hepatitis $\mathrm{B}$ virus; $\mathrm{HCC}$, hepatocellular carcinoma; HCV, hepatitis C virus; HDL, high-density lipoprotein; HEK, human embryonic kidney; HNF, hepatocyte nuclear factor; HO-1, heme oxygenase-1; IFN $\beta$, interferon $\beta$; Igf1R, insulin-like growth factor 1 receptor; IRES, internal ribosome entry site; IRGs, IFN-regulated genes; LDL, low-density lipoprotein; LNA, locked nucleic acid; miRNA, microRNA; NCR, noncoding region; PNR, primary nonresponders; RISC, RNA-induced silencing complex; SRF, serum response factor; UTR, untranslated regions

\section{REFERENCES}

Bai, S., Nasser, M.W., Wang, B., Hsu, S.H., Datta, J., Kutay, H., Yadav, A., Nuovo, G., Kumar, P., and Ghoshal, K. (2009). Mi-
croRNA-122 inhibits tumorigenic properties of hepatocellular carcinoma cells and sensitizes these cells to sorafenib. J Biol Chem 284, 32015-32027.

Belver, L., de Yébenes, V.G., and Ramiro, A.R. (2010). MicroRNAs prevent the generation of autoreactive antibodies. Immunity 33 , 713-722.

Burchard, J., Zhang, C., Liu, A.M., Poon, R.T., Lee, N.P., Wong, K.F., Sham, P.C., Lam, B.Y., Ferguson, M.D., Tokiwa, G., et al. (2010). microRNA-122 as a regulator of mitochondrial metabolic gene network in hepatocellular carcinoma. Mol Syst Biol 6, 1-12.

Burns, D.M., D’Ambrogio, A., Nottrott, S., and Richter, J.D. (2011). CPEB and two poly(A) polymerases control miR-122 stability and p53 mRNA translation. Nature 473, 105-108.

Carissimi, C., Fulci, V., and Macino, G. (2009). MicroRNAs: novel regulators of immunity. Autoimmun Rev 8, 520-524.

Chang, J., Guo, J.T., Jiang, D., Guo, H., Taylor, J.M., and Block, T.M. (2008). Liver-specific microRNA miR-122 enhances the replication of hepatitis $C$ virus in nonhepatic cells. J Virol 82, 8215-8223.

Chen, S., Ni, M., Yu, B., Lv, T., Lu, M., and Gong, F. (2007). Construction and identification of a human liver specific microRNA eukaryotic expression vector. Cell Mol Immunol 4, 473-477.

Chen, Y., Shen, A., Rider, P.J., Yu, Y., Wu, K., Mu, Y., Hao, Q., Liu, Y., Gong, H., Zhu, Y., et al. (2011). A liver-specific microRNA binds to a highly conserved RNA sequence of hepatitis $B$ virus and negatively regulates viral gene expression and replication. FASEB J 25, 4511-4521.

Coulouarn, C., Factor, V.M., Andersen, J.B., Durkin, M.E., and Thorgeirsson, S.S. (2009). Loss of miR-122 expression in liver cancer correlates with suppression of the hepatic phenotype and gain of metastatic properties. Oncogene 28, 3526-3536.

Díaz-Toledano, R., Ariza-Mateos, A., Birk, A., Martínez-García, B., and Gómez, J. (2009). In vitro characterization of a miR-122-sensitive double-helical switch element in the $5^{\prime}$ region of hepatitis C virus RNA. Nucleic Acids Res 37, 5498-5510.

Elmén, J., Lindow, M., Schütz, S., Lawrence, M., Petri, A., Obad, S., Lindholm, M., Hedtjärn, M., Hansen, H.F., Berger, U., et al. (2008a). LNA-mediated microRNA silencing in non-human primates. Nature 452, 896-899.

Elmén, J., Lindow, M., Silahtaroglu, A., Bak, M., Christensen, M., Lind-Thomsen, A., Hedtjärn, M., Hansen, J.B., Hansen, H.F., Straarup, E.M., et al. (2008b). Antagonism of microRNA-122 in mice by systemically administered LNA-antimiR leads to up-regulation of a large set of predicted target mRNAs in the liver. Nucleic Acids Res 36, 1153-1162.

Esau, C., Davis, S., Murray, S.F., Yu, X.X., Pandey, S.K., Pear, M., Watts, L., Booten, S.L., Graham, M., McKay, R., et al. (2006). miR-122 regulation of lipid metabolism revealed by in vivo antisense targeting. Cell Metab 3, 87-98.

Esau, C.C. (2008). Inhibition of microRNA with antisense oligonucleotides. Methods 44, 55-60.

Fan, C.G., Wang, C.M., Tian, C., Wang, Y., Li, L., Sun, W.S., Li, R.F., and Liu, Y.G. (2011). miR-122 inhibits viral replication and cell proliferation in hepatitis $B$ virus-related hepatocellular carcinoma and targets NDRG3. Oncol Rep 26, 1281-1286.

Filipowicz, W., and Grosshans, H. (2011). The liver-specific microRNA miR-122: biology and therapeutic potential. Prog Drug Res 67, 221-238.

Fire, A., Xu, S., Montgomery, M.K., Kostas, S.A., Driver, S.E., and 
Mello, C.C. (1998). Potent and specific genetic interference by double-stranded RNA in Caenorhabditis elegans. Nature 391, 806-811.

Fornari, F., Gramantieri, L., Giovannini, C., Veronese, A., Ferracin, M., Sabbioni, S., Calin, G.A., Grazi, G.L., Croce, C.M., Tavolari, S., et al. (2009). MiR-122/cyclin G1 interaction modulates p53 activity and affects doxorubicin sensitivity of human hepatocarcinoma cells. Cancer Res 69, 5761-5767.

Girard, M., Jacquemin, E., Munnich, A., Lyonnet, S., and Henrion-Caude, A. (2008). miR-122, a paradigm for the role of microRNAs in the liver. J Hepatol 48, 648-656.

Henke, J.I., Goergen, D., Zheng, J., Song, Y., Schüttler, C.G., Fehr, C., Jünemann, C., and Niepmann, M. (2008). microRNA-122 stimulates translation of hepatitis C virus RNA. EMBO J 27, 3300-3310.

Jangra, R.K., Yi, M., and Lemon, S.M. (2010). Regulation of hepatitis $C$ virus translation and infectious virus production by the microRNA miR-122. J Virol 84, 6615-6625.

Jopling, C.L., Yi, M., Lancaster, A.M., Lemon, S.M., and Sarnow, P. (2005). Modulation of hepatitis C virus RNA abundance by a liver-specific MicroRNA. Science 309, 1577-1581.

Karakatsanis A, Papaconstantinou I, Gazouli M, Lyberopoulou A, Polymeneas G, Voros D. (2011). Expression of microRNAs, miR-21, miR-31, miR-122, miR-145, miR-146a, miR-200c, miR-221, miR-222, and miR-223 in patients with hepatocellular carcinoma or intrahepatic cholangiocarcinoma and its prognostic significance. Mol Carcinog 27 Dec 2011. doi: 10.1002/mc.21864

Kasinski, A.L., and Slack, F.J. (2011). Epigenetics and genetics. MicroRNAs en route to the clinic: progress in validating and targeting microRNAs for cancer therapy. Nat Rev Cancer 11, 849-864.

Krützfeldt, J., Rajewsky, N., Braich, R., Rajeev, K.G., Tuschl, T., Manoharan, M., and Stoffel, M. (2005). Silencing of microRNAs in vivo with 'antagomirs'. Nature 438, 685-689.

Kummee, P., Tangkijvanich, P., Poovorawan, Y., and Hirankarn, N. (2007). Association of HLA-DRB1*13 and TNF-alpha gene polymorphisms with clearance of chronic hepatitis $B$ infection and risk of hepatocellular carcinoma in Thai population. J Viral Hepat 14, 841-848.

Lanford, R.E., Hildebrandt-Eriksen, E.S., Petri, A., Persson, R., Lindow, M., Munk, M.E., Kauppinen, S., and Ørum, H. (2010). Therapeutic silencing of microRNA-122 in primates with chronic hepatitis C virus infection. Science 327, 198-201.

Laudadio, I., Manfroid, I., Achouri, Y., Schmidt, D., Wilson, M.D., Cordi, S., Thorrez, L., Knoops, L., Jacquemin, P., Schuit, F., et al. (2012). A feedback loop between the liver-enriched transcription factor network and miR-122 controls hepatocyte differentiation. Gastroenterology 142, 119-129.

Lewis, B.P., Burge, C.B., and Bartel, D.P. (2005). Conserved seed pairing, often flanked by adenosines, indicates that thousands of human genes are microRNA targets. Cell 120, 15-20.

Lindsay, M.A. (2008). microRNAs and the immune response. Trends Immunol 29, 343-351.

Luo, X., Yang, W., Ye, D.Q., Cui, H., Zhang, Y., Hirankarn, N., Qian, X., Tang, Y., Lau, Y.L., de Vries, N., et al. (2011). A functional variant in microRNA-146a promoter modulates its expression and confers disease risk for systemic lupus erythematosus. PLoS Genet 7, e1002128.

Ma, L., Liu, J., Shen, J., Liu, L., Wu, J., Li, W., Luo, J., Chen, Q., and
Qian, C. (2010). Expression of miR-122 mediated by adenoviral vector induces apoptosis and cell cycle arrest of cancer cells. Cancer Biol Therapy 9, 554-561.

Miyoshi, K., Okada, T.N., Siomi, H., and Siomi, M.C. (2009). Characterization of the miRNA-RISC loading complex and miRNA-RISC formed in the Drosophila miRNA pathway. RNA 15, 1282-1291.

Mizuguchi, Y., Mishima, T., Yokomuro, S., Arima, Y., Kawahigashi, Y., Shigehara, K., Kanda, T., Yoshida, H., Uchida, E., Tajiri, T., et al. (2011). Sequencing and bioinformatics-based analyses of the microRNA transcriptome in hepatitis B-related hepatocellular carcinoma. PLoS One 6, e15304.

Moore, K.J., Rayner, K.J., Suárez, Y., and Fernández-Hernando, C. (2010). microRNAs and cholesterol metabolism. Trends Endocrinol Metab 21, 699-706.

Morita, K., Taketomi, A., Shirabe, K., Umeda, K., Kayashima, H., Ninomiya, M., Uchiyama, H., Soejima, Y., and Maehara, Y. (2011). Clinical significance and potential of hepatic microRNA-122 expression in hepatitis C. Liver Int 31, 474-484.

Murchison, E.P., Stein, P., Xuan, Z., Pan, H., Zhang, M.Q., Schultz, R.M., and Hannon, G.J. (2007). Critical roles for Dicer in the female germline. Genes Dev 21, 682-693.

Narbus, C.M., Israelow, B., Sourisseau, M., Michta, M.L., Hopcraft, S.E., Zeiner, G.M., and Evans, M.J. (2011). HepG2 cells expressing microRNA miR-122 support the entire hepatitis $C$ virus life cycle. J Virol 85, 12087-12092.

Newman, M.A., and Hammond, S.M. (2010). Emerging paradigms of regulated microRNA processing. Genes Dev 24, 1086-1092.

Norman, K.L., and Sarnow, P. (2010). Modulation of hepatitis C virus RNA abundance and the isoprenoid biosynthesis pathway by microRNA miR-122 involves distinct mechanisms. J Virol 84, 666-670.

Pang, P.S., Pham, E.A., Elazar, M., Patel, S.G., Eckart, M.R., and Glenn, J.S. (2012). Structural map of a Pan PS, microRNA-122: Hepatitis C virus complex. J Virol 86, 1250-1254.

Pedersen, I.M., Cheng, G., Wieland, S., Volinia, S., Croce, C.M., Chisari, F.V., and David, M. (2007). Interferon modulation of cellular microRNAs as an antiviral mechanism. Nature 449, 919-922.

Ramezani, A., Hasanjani Roshan, M.R., Kalantar, E., Eslamifar, A., Banifazl, M., Taeb, J., Aghakhani, A., Gachkar, L., and Velayati, A.A. (2008). Association of human leukocyte antigen polymorphism with outcomes of hepatitis B virus infection. J Gastroenterol Hepatol 23, 1716-1721.

Reinhart, B.J., Slack, F.J., Basson, M., Pasquinelli, A.E., Bettinger, J.C., Rougvie, A.E., Horvitz, H.R., and Ruvkun, G. (2000). The 21-nucleotide let-7 RNA regulates developmental timing in Caenorhabditis elegans. Nature 403, 901-906.

Rusca, N., and Monticelli, S. (2011). MiR-146a in Immunity and Disease. Mol Biol Int 2011, 437301.

Sage, J., Ventura, A., and Macino, G. (2011). miR than meets the eye. Genes Dev 25, 1663-1667.

Sarasin-Filipowicz, M., Krol, J., Markiewicz, I., Heim, M.H., and Filipowicz, W. (2009). Decreased levels of microRNA miR-122 in individuals with hepatitis $C$ responding poorly to interferon therapy. Nat Med 15, 31-33.

Shan, Y., Zheng, J., Lambrecht, R.W., and Bonkovsky, H.L. (2007). Reciprocal effects of micro-RNA-122 on expression of heme oxygenase- 1 and hepatitis $C$ virus genes in human hepatocytes. 
Gastroenterology 133, 1166-1174.

Shimakami, T., Yamane, D., Jangra, R.K., Kempf, B.J., Spaniel, C., Barton, D.J., and Lemon, S.M. (2012). Stabilization of hepatitis C virus RNA by an Ago2-miR-122 complex. Proc Natl Acad Sci U S A 109, 941-946.

Starczynowski, D.T., Kuchenbauer, F., Argiropoulos, B., Sung, S., Morin, R., Muranyi, A., Hirst, M., Hogge, D., Marra, M., Wells, R.A., et al. (2010). Identification of miR-145 and miR-146a as mediators of the $5 q$ - syndrome phenotype. Nat Med 16, 49-58.

Tan, A.T., Loggi, E., Boni, C., Chia, A., Gehring, A.J., Sastry, K.S., Goh, V., Fisicaro, P., Andreone, P., Brander, C., et al. (2008). Host ethnicity and virus genotype shape the hepatitis $B$ virus-specific T-cell repertoire. J Virol 82, 10986-10997.

Wang S, Qiu L, Yan X, Jin W, Wang Y, Chen L, Wu E, Ye X, Gao GF, Wang F, Chen Y, Duan Z, Meng S. (2012). Loss of MiR-122 expression in patients with hepatitis $B$ enhances hepatitis $B$ virus replication through cyclin G1 modulated P53 activity. Hepatology 23 Feb 2012. doi: 10.1002/hep.24809

Xu, H., He, J.H., Xiao, Z.D., Zhang, Q.Q., Chen, Y.Q., Zhou, H., and Qu, L.H. (2010). Liver-enriched transcription factors regulate microRNA-122 that targets CUTL1 during liver development. Hepatology 52, 1431-1442.

Xu, J., Zhu, X. , et al. (2012). MicroRNA-122 suppresses cell proliferation and induces cell apoptosis in hepatocellular carcinoma by directly targeting Wnt/beta-catenin pathway. Liver Int 26 Jan 2012. doi: 10.1111/j.1478-3231.2011.02750.x

Xu, Y., Xia, F., Ma, L., Shan, J., Shen, J., Yang, Z., Liu, J., Cui, Y., Bian, X., Bie, P., et al. (2011). MicroRNA-122 sensitizes HCC cancer cells to adriamycin and vincristine through modulating expression of MDR and inducing cell cycle arrest. Cancer Lett 310, 160-169.

Yang, F., Zhang, L., Wang, F., Wang, Y., Huo, X.S., Yin, Y.X., Wang, Y.Q., Zhang, L., and Sun, S.H. (2011). Modulation of the unfolded protein response is the core of microRNA-122-involved sensitivity to chemotherapy in hepatocellular carcinoma. Neoplasia 13, 590-600.

Zeng, C., Wang, R., Li, D., Lin, X.J., Wei, Q.K., Yuan, Y., Wang, Q., Chen, W., and Zhuang, S.M. (2010). A novel GSK-3 beta-C/EBP alpha-miR-122-insulin-like growth factor 1 receptor regulatory circuitry in human hepatocellular carcinoma. Hepatology 52, 1702-1712.

Zhang, L., Zhang, B., Valdez, J.M., Wang, F., Ittmann, M., and Xin, L. (2010). Dicer ablation impairs prostate stem cell activity and causes prostate atrophy. Stem Cells 28, 1260-1269.

Zhou, X., Jeker, L.T., Fife, B.T., Zhu, S., Anderson, M.S., McManus, M.T., and Bluestone, J.A. (2008). Selective miRNA disruption in T reg cells leads to uncontrolled autoimmunity. J Exp Med 205, 1983-1991. 